\title{
MULTIDISCIPLINARY TREATMENT OF PEDIATRIC MIGRAINE
}

The long-term effectiveness and outcome of multidisciplinary treatment of childhood headaches were evaluated at 1,2, and 5 years at the Cincinnati Children's Hospital Medical Center, OH. Diagnosis was based on clinical impression and ICHD criteria. Data on severity, frequency, duration, participation in activities during headaches, effect of treatment, and school days missed were collected from clinic follow-up questionnaire or via telephone. The team-based treatment plan included acute, prophylactic, behavioral therapy, and teaching. Bio-behavioral therapy included changes in diet, hydration, sleep pattern, and activity. Days of school missed were used as a disability measure; a marked decrease occurred from $4.5+/$ 9.5 at initial visit to $1.55+/-2.8$ at 5 years $(\mathrm{p}<0.001)$. Headache frequency was $13.4 /$ month initially, 4.9 at 1 year, 4.7 at 2 years, and 4.5 at 5 years $(p<0.001)$. Headache severity was also significantly decreased. Patients with less frequent and shorter duration headaches at initial visit were less likely to return for follow-up, but continued to do well at 5-year assessment. (Kabbouche MA, Powers SW, Vockell A-LB et al. Outcome of a multidisciplinary approach to pediatric migraine at 1, 2, and 5 years. Headache Nov 2005;45:1298-1303). (Respond: Dr Marielle A Kabbouche, Cincinnati Children's Hospital Medical Center, Headache Center, Division of Neurology, MLC \#2015, 3333 Burnet Ave, Cincinnati, OH 45229).

COMMENT. Children with more severe migraine headaches at initial presentation should benefit from a regular multidisciplinary treatment plan.

Topiramate was effective in reducing the frequency and severity of chronic daily headache in $62 \%$ of 21 patients, ages 6-18 years, using a low dose (average 30/mg/day). (Borzy JC, Koch TK, Schimschock JR. Pediatr Neurol Nov 2005;33:314-316).

\section{TENSION-TYPE AND OTHER PRIMARY HEADACHES}

A review of the literature on tension-type and other "nonmigrainous" primary headaches is presented from Children's Hospital of the King's Daughters, Eastern Virginia Medical School, Norfolk, VA. Tension-type headaches are mild to moderate in intensity, often frontal in location, duration minutes to hours, and lack autonomic features. Other primary headaches and neuralgias are brief, with or without autonomic symptoms. They include cluster headaches, paroxysmal hemicrania responsive to indomethacin, short-lasting unilateral neuralgiform headache with conjunctival injection and tearing (SUNCT), stabbing headache, cough headache, exertional, headache with sexual activity, hypnic (alarm clock) headache, thunderclap headache, cranial neuralgias, trigeminal neuralgia, glossopharyngeal neuralgia, occipital neuralgia, and ice cream headache, Cluster headaches, paroxysmal hemicrania, and SUNCT are similar in location (unilateral, orbital, and supraorbital), their duration differs widely from 15-180 minutes for cluster headaches, 2-30 min for paroxysmal hemicrania, and 5-240 seconds for SUNCT, the frequency of attacks is $8 /$ day, $5 /$ day, and 3200/day, respectively, and a response to indomethacin occurs only for paroxysmal hemicrania. (Lewis DW, Gozzo YF, Avner MT. The "other" primary headaches in children and adolescents. Pediatr Neurol Nov 2005;33:303-313). (Respond: Dr Lewis, 850 Southampton Ave, Norfolk, VA 23510). 
COMMENT. The prevalence of tension-type headache (TTH) in children and adolescents has been estimated variously from $11 \%$ to $73 \%$. In one large series of 8255 adolescents in Norway, a 1-year-prevalence of TTH was $18 \%$, compared to $7 \%$ for migraine (Zwart J et al. Cephalalgia 2004;24:373-379 - cited by above authors). Since the differentiation of TTH and migraine is questionable, except in terms of severity, treatment strategies are similar. Other primary headaches listed above would require more specific therapies.

\section{ATTENTION DEFICIT AND LEARNING DISORDERS}

\section{FUNCTIONAL MRI AND ATTENTION DEFICIT HYPERACTIVITY DISORDER}

The Stroop effect, a measure for selective attention, on behavioral and brain activation of attention deficit hyperactivity disorder (ADHD) children ( 9 boys, ages $9.8-14.5$ years, off or on methylphenidate) and 9 controls was studied using event-related functional magnetic resonance imaging (fMRI) at the Institute of Mental Health, Peking University, Beijing, China, and other centers in China and at Harvard Medical School, Boston, MA, USA. The Stroop effect (reaction time in IC [interference condition with high cognitive load] longer than in NC [neutral condition and low cognitive load]) was present in the behavioral performance of control children, but was absent in untreated ADHD children, not receiving MPH. When MPH was administered to ADHD children a Stroop effect was obtained. The activation volume (AV) of prefrontal cortex (PFC) in both the neutral $(\mathrm{NC})$ and interference conditions (IC) in ADHD children off MPH was smaller than in controls, indicating frontal lobe hypofunction in either high (IC) or low (NC) cognitive load. The anterior cingulate cortex (ACC), basal ganglia (BG), insula and cerebellum showed hypofunction in high cognitive load (IC). In low cognitive load (NC), the ACC was normal, while the BG, insula and cerebellum showed compensatory hyperfunction, suggesting a compensatory neural network when ADHD children have a lower cognitive load. (Zang Y-F, Jin Z, Weng X-C et al. Functional MRI in attention-deficit hyperactivity disorder: evidence for hypofrontality. Brain \& Dev December 2005;27:544-550). (Respond: Dr Yu-Feng Wang, Institute of Mental Health, Peking University, Huayuanbeilu 51, Haidian Districk, Beijing 100083, China).

COMMENT. These findings confirm previous reports of hypofrontality in ADHD adults, using PET studies and cerebral glucose metabolism (Zametkin AJ et al. $\mathbf{N}$ Engl $\mathbf{J}$ Med 1990;323:1361-6). A compensatory network involving basal ganglia and cerebellum may be implicated, when ADHD children are presented with lower cognitive load tasks. Functional MRI studies should offer an "in vivo" window, not only to brain regions that control cognitive and behavioral functions in ADHD, but also to the effect of medications on the circuits involved in attention ((Curatolo P. Editorial. The neurology of attention deficit/hyperactivity disorder. Brain \& Dev Dec 2005;27:541-543). 